\title{
B7-H3 regulates KIF15-activated ERK1/2 pathway and contributes to radioresistance in colorectal cancer
}

\author{
Yanchao Ma ${ }^{1,2}$, Shenghua Zhan ${ }^{3}$, Huimin Lu', Ruoqin Wang ${ }^{1}$, Yunyun Xu', Guangbo Zhang ${ }^{3,5}$, Lei Cao ${ }^{2}$, \\ Tongguo Shi $\mathbb{B}^{3,2}$, Xueguang Zhang ${ }^{2}$ and Weichang Chen ${ }^{1,5}$
}

\begin{abstract}
As an important modality for the local control of colorectal cancer (CRC), radiotherapy or neoadjuvant radiotherapy is widely applied in the clinic, but radioresistance has become a major obstacle for CRC radiotherapy. Here we reported that $\mathrm{B} 7-\mathrm{H} 3$, an important costimulatory molecule, is associated with radioresistance in CRC. The expression of B7-H3 was obviously increased in CRC cells after irradiation. The enhanced expression of B7-H3 promoted, while the knockdown of B7-H3 inhibited, colony formation and cell activity in CRC cells following radiation treatment. B7-H3 overexpression reduced S phase arrest and protected cell apoptosis induced by radiation, whereas B7-H3 knockdown had the opposite effects. In addition, B7-H3 blockade by 3E8, a specific B7-H3 antibody, significantly sensitized CRC cells to irradiation in vivo. Mechanistic analysis revealed that B7-H3 regulated KIF15 via RNA sequencing, which was in dependent of NF-KB pathway. And small interfering RNA (siRNA)-mediated KIF15 silencing or KIF15 blockade by the inhibitor SB743921 abolished the effect of B7-H3 on radioresistance in vitro and in vivo. Similar to B7-H3, we find that the protein expression levels of KIF15, which showed a positive correlation with B7-H3, was abnormal upregulated in cancer tissues than in adjacent normal tissues and associated with TNM stage. Finally, B7-H3/KIF15 enhanced resistance against irradiation in CRC cells via activating ERK1/2 signaling, a key pathway involved in radioresistance in cancer. Our findings reveal an alternative mechanism by which CRC cells can acquire radioresistance via the B7-H3/ KIF15/ERK axis.
\end{abstract}

\section{Introduction}

Colorectal cancer (CRC) is the third most commonly occurring malignancy and accounts for more than $9 \%$ of all cancer-related deaths worldwide ${ }^{1}$. Neoadjuvant radiotherapy/chemoradiotherapy (neoRT/CRT) following surgery has been approved by NCCN and is required for comprehensive therapy for locally advanced stage II and

\footnotetext{
Correspondence: Tongguo Shi (shitg@suda.edu.cn) or

Xueguang Zhang (xueguangzh@126.com) or

Weichang Chen (weichangchen@126.com)

'Department of Gastroenterology, The First Affiliated Hospital of Soochow

University, 188 Shizi Road, Suzhou, China

${ }^{2}$ Jiangsu Key Laboratory of Clinical Immunology, Soochow University, 708

Renmin Road, Suzhou, China

Full list of author information is available at the end of the article

Edited by T. Brunner
}

III $\mathrm{CRC}^{2}$. In addition, preoperative chemoradiotherapy combined with surgery could improve the locoregional control of CRC. However, the treatment effect of both neoRT/CRT and preoperative chemoradiotherapy is still unsatisfactory because of chemoresistance and radioresistance $^{3}$. Importantly, approximately $50 \%$ of CRC patients experience recurrence and metastasis after radiotherapy ${ }^{4}$. Therefore, there is an urgent need to understand the mechanisms of radioresistance and identify biomarkers that predict radioresistance in CRC patients.

As a type I transmembrane protein, $\mathrm{B} 7-\mathrm{H} 3$ belongs to the important immune checkpoint B7 ligand family, which provides a costimulatory signal for $\mathrm{T}$-cells in the tumor microenvironment and promotes tumor

\section{(c) The Author(s) 2020}

(c) (i) Open Access This article is licensed under a Creative Commons Attribution 4.0 International License, which permits use, sharing, adaptation, distribution and reproduction cc) in any medium or format, as long as you give appropriate credit to the original author(s) and the source, provide a link to the Creative Commons license, and indicate if changes were made. The images or other third party material in this article are included in the article's Creative Commons license, unless indicated otherwise in a credit line to the material. If material is not included in the article's Creative Commons license and your intended use is not permitted by statutory regulation or exceeds the permitted use, you will need to obtain permission directly from the copyright holder. To view a copy of this license, visit http://creativecommons.org/licenses/by/4.0/. 
progression and escape ${ }^{5-7}$. Many studies have suggested that the aberrant expression of B7-H3 exists in different cancer types, including pancreatic carcinoma ${ }^{8}$, esophageal carcinoma ${ }^{9}$, hepatocellular cancer ${ }^{10}$, colorectal cancer ${ }^{11}$, non-small cell lung cancer ${ }^{12}$ and gastric cancer ${ }^{13}$. The frequency of B7-H3-positive circulating epithelial tumor cells (CETCs) is significantly higher in breast cancer patients who receive radiotherapy than in patients who do not receive irradiation, suggesting that the upregulation of B7-H3 expression on CETCs could be a possible mechanism of acquired radioresistance in breast cancer patients ${ }^{14}$. Nevertheless, the functional roles and underlying signaling cascades of B7-H3 associated with radioresistance in CRC have yet to be investigated.

Herein, we first demonstrated that the expression of B7$\mathrm{H} 3$ was obviously increased in CRC cells after irradiation. Further in vitro and in vivo functional analyses showed that $\mathrm{B} 7-\mathrm{H} 3$ enhanced resistance against irradiation in CRC cells by upregulating KIF15 expression via NF- $\mathrm{kB}$, which activated ERK1/2 signaling, a key pathway involved in radioresistance in cancers ${ }^{15}$. B7-H3 blockade by $3 \mathrm{E} 8$, a specific B7-H3 antibody ${ }^{16}$, significantly sensitized CRC cells to irradiation in vivo. Moreover, the expression of B7-H3 was positively correlated with KIF15 expression in CRC tissue samples. Overall, our data suggest that B7-H3 contributes to $\mathrm{CRC}$ radioresistance and that targeting this molecule may be beneficial for CRC treatment.

\section{Materials and methods}

\section{Cell lines and cell culture}

NCM460, HCT8, HT29, SW480, SW620, HCT116 and RKO CRC cell lines were purchased from the Chinese Academy of Science Cell Bank and cultured in DMEM and RPMI-1640 medium (BioInd, Beit Haemek, Israel) including $10 \%$ fetal bovine serum (FBS, BioInd), $100 \mathrm{U} / \mathrm{ml}$ penicillin and $100 \mathrm{mg} / \mathrm{ml}$ streptomycin (Gibco, Grand Island, USA) in a humidified atmosphere of $5 \% \mathrm{CO}_{2}$ at $37^{\circ} \mathrm{C}$.

\section{Immunohistochemistry}

Sections from paraffin-embedded tissues were incubated with a goat anti-human B7-H3 antibody (1:200, R\&D Systems, \#AF1027), a rabbit anti-human KIF15 antibody (1:2000, Proteintech, \#55407-1-AP) or a rabbit anti-human Ki67 antibody (1:500, Abcam, ab15580) overnight at $4{ }^{\circ} \mathrm{C}$. This step was followed by staining (45 $\mathrm{min}$ at room temperature) with the corresponding HRP-labeled rabbit anti-goat secondary antibody or goat anti-rabbit secondary antibody (Invitrogen). Next, the sections were visualized by staining with 3,3'-diaminobenzidine (Biocare Medical, CA, USA) and counterstaining with hematoxylin (Sigma). The numbers of Ki67positive cells and total cells were analyzed using a microscope (Leica, Buffalo Grove, USA).
All sections were then reviewed blindly by two experienced pathologists (Dr. Cao and Dr. Zhan). The scoring criteria for B7-H3 and KIF15 immunostaining were based on clinical data and adopted the semiquantitative immunoreactive score (IRS) system ${ }^{17}$. Briefly, category A (intensity of immunostaining) was scored using the following criteria: 0 , negative; 1 , weak; 2 , moderate; and 3 , strong. Category B (percentage of immunoreactive cells) was scored using the following criteria: 1 (0-25\%); 2 (26-50\%); 3 (51-75\%); and 4 (76-100\%). Final scores were calculated by multiplying the scores of categories A and $B$ in the same section; the scores ranged from 0 to 12 .

\section{Statistical analysis}

All statistical analyses were performed using GraphPad 6.0 statistical software packages. Statistically significant differences between groups were determined using Student's $t$-test. A $P$-value of $<0.05$ was considered statistically significant in all cases.

\section{Other methods}

Detailed description of other methods used in this study are provided in Supplementary Materials and Methods.

\section{Results \\ B7-H3 enhances the radioresistance of CRC cells in vitro}

As shown in Supplementary Fig. S1a, B7-H3 was frequently upregulated in the CRC cell lines (RKO, HCT116, HCT8, HT29, SW480 and SW620) compared to the human colon healthy cell line (NCM460), suggesting B7$\mathrm{H} 3$ overexpression has a crucial role in CRC progression. To better understand the link between $\mathrm{B} 7-\mathrm{H} 3$ and CRC radioresistance, we first investigated the expression of $\mathrm{B} 7$ $\mathrm{H} 3$ in CRC cells after ionizing radiation (IR). As shown in Fig. 1a, b, both the mRNA and protein levels of B7-H3 were significantly increased in CRC cells after IR. To determine whether $\mathrm{B} 7-\mathrm{H} 3$ mediated radioresistance in CRC cells, we overexpressed and knocked down B7-H3 in CRC cells. RT-qPCR and Western blot further confirmed that B7-H3 expression was significantly increased in stable B7-H3-overexpressing CRC cell lines (Supplementary Fig. $\mathrm{S} 1 \mathrm{~b}, \mathrm{c}$ ), while the B7-H3 level was obviously decreased in stable B7-H3-knockdown CRC cell lines (Supplementary Fig. S1d, e). Next, we noted that B7-H3-overexpressing CRC cells had a significant enhancement in colony formation after IR (SER $=0.67$ for B7-H3-overexpressing HCT116 cells and SER $=0.78$ for B7-H3-overexpressing RKO cells, Fig. 1c). In contrast, B7-H3-knockdown CRC cells showed significantly fewer colony numbers than their respective scramble control cells after 4 Gy X-ray irradiation (Supplementary Fig. S1f). In addition, cell viability analysis showed that $\mathrm{B} 7-\mathrm{H} 3$ overexpression enhanced the radioresistance of $\mathrm{CRC}$ cells (Fig. 1d), while $\mathrm{B} 7-\mathrm{H} 3$ 


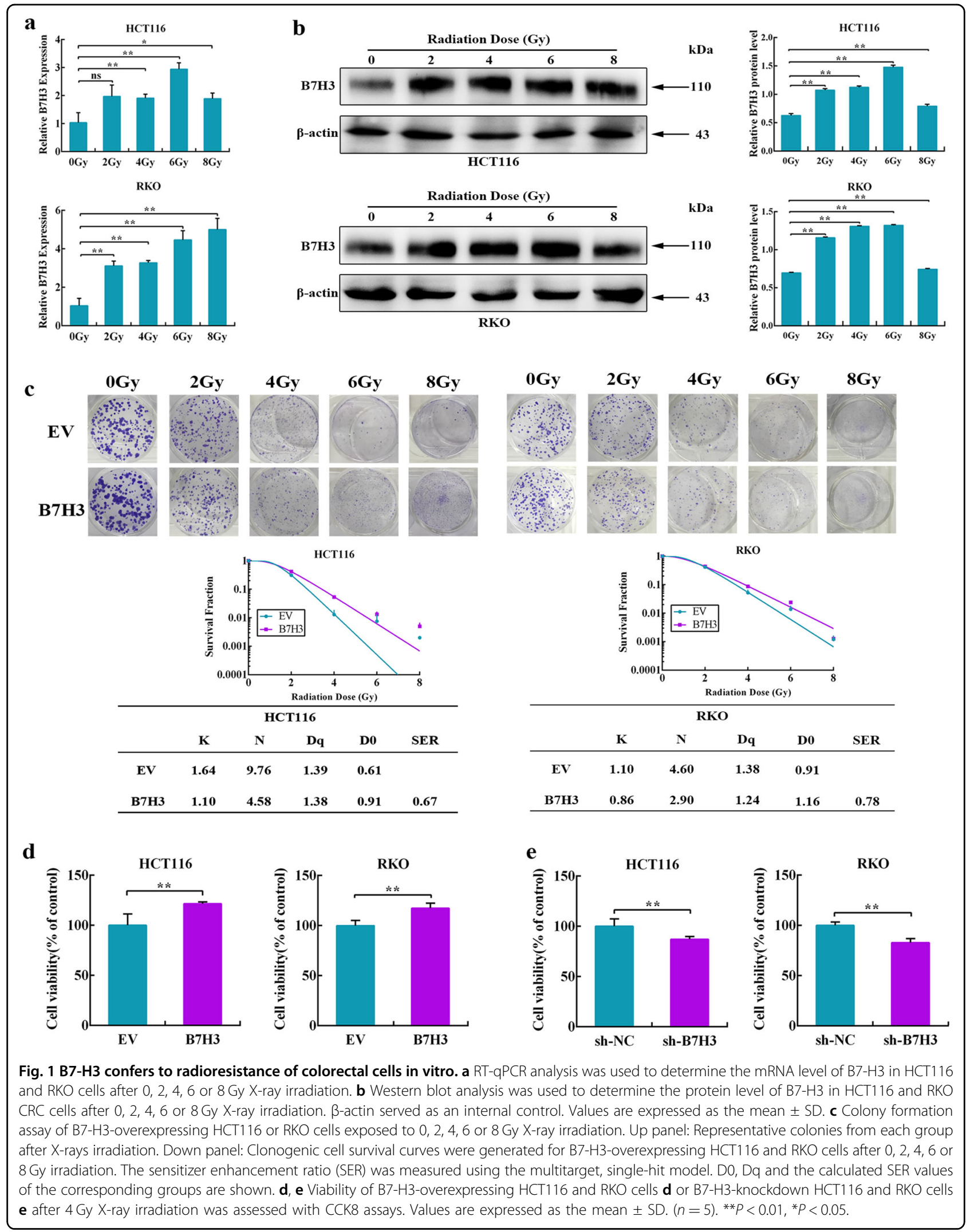


knockdown significantly reduced the viability of CRC cells (Fig. 1e).

To investigate whether $\mathrm{B} 7-\mathrm{H} 3$-associated radioresistance was related to cell cycle progression, the cell cycle distribution of B7-H3-overexpressing or B7-H3knockdown CRC cells after 4Gy X-ray irradiation was analyzed by flow cytometry. The results showed that the percentage of B7-H3-overexpressing CRC cells in S phase was much lower than that of control cells (Fig. 2a). In contrast, the percentage of B7-H3-knockdown CRC cells in S phase was significantly higher than that of sh$\mathrm{NC}$ cells (Fig. 2b). Moreover, the protein levels of Cyclin B1 and CDK1, two cell cycle-associated genes, were examined in B7-H3-overexpressing or B7-H3-knockdown CRC cells by Western blot. B7-H3-overexpressing reduced, whereas $\mathrm{B} 7-\mathrm{H} 3$ knockdown induced, the expression of CDK1 in CRC cells after 4 Gy X-ray irradiation (Supplementary Fig. S2a, b). However, B7-H3 had no effect on the Cyclin B1 expression (Supplementary Fig. S2a, b).
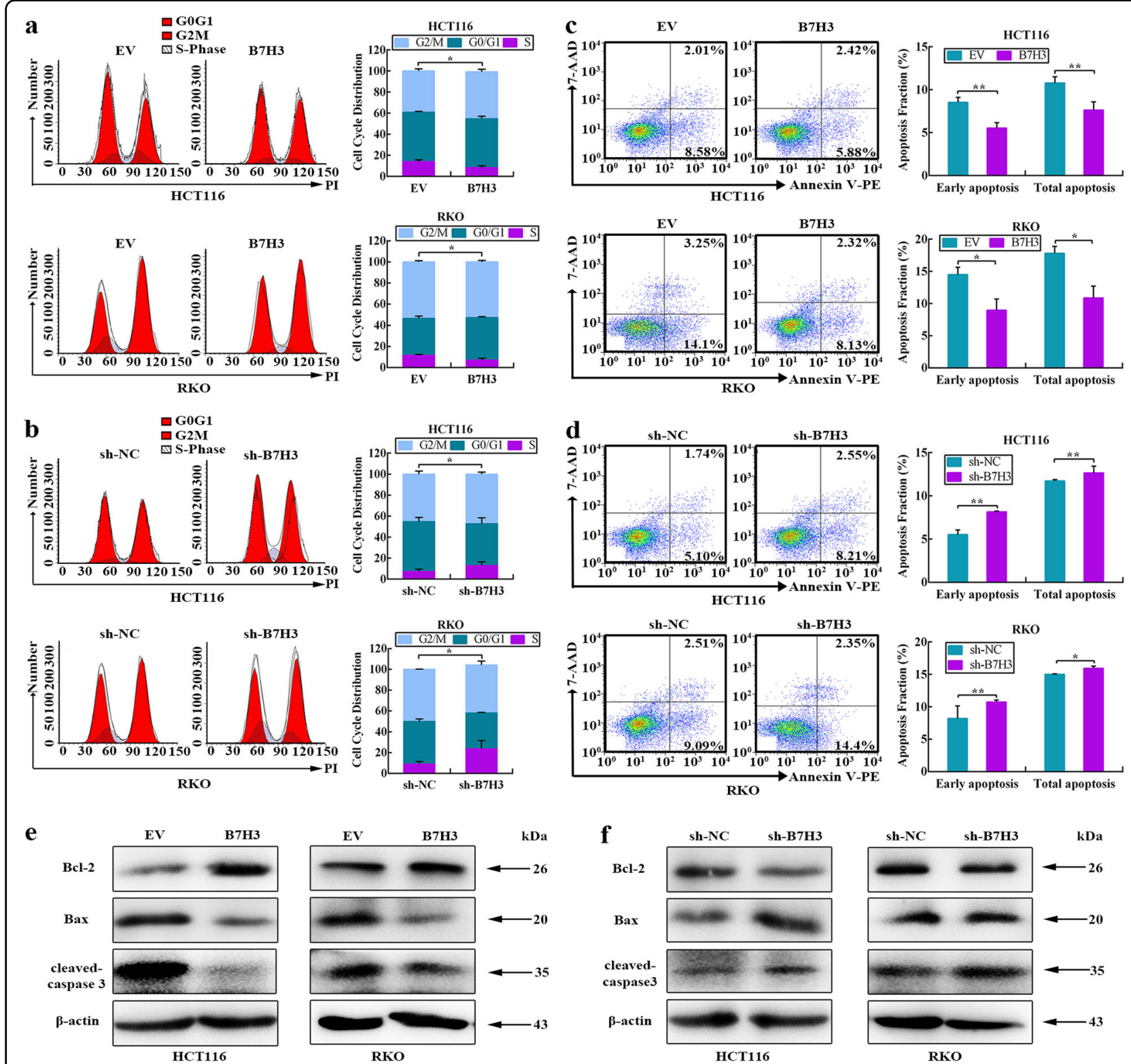

Fig. 2 B7-H3 reduces S phase arrest and cell apoptosis of colorectal cells. $\mathbf{a}$, $\mathbf{b}$ The effect of B7-H3 overexpression (a) and B7-H3 knockdown (b) on the cell cycle distribution in HCT116 and RKO cells after exposure to 4 Gy X-ray irradiation. $\mathbf{c}$, d Apoptosis was measured using Annexin V/7-AAD double staining in B7-H3-overexpressing (c) or B7-H3-knockdown (d) CRC cells after exposure to 4 Gy X-ray irradiation. The data are shown as the mean \pm SD of three independent experiments. e, $\mathbf{f}$ The protein expression of $\mathrm{BCl}-2$, Bax and cleaved-caspase 3 in B7-H3-overexpressing (e) or B7-H3knockdown (f) CRC cells after exposure to 4 Gy $X$-ray irradiation. $\beta$-actin served as a loading control. ${ }^{*} P<0.01,{ }^{*} P<0.05$. 
Next, we used Annexin V/7-AAD double-staining assays to determine the effects of B7-H3 on apoptosis in CRC cells after $4 \mathrm{~Gy} \mathrm{X}$-ray irradiation. The results showed that $\mathrm{B} 7-\mathrm{H} 3$ overexpression decreased both the early and total apoptotic populations of CRC cells compared with EV cells after 4 Gy X-ray irradiation (Fig. 2c). By contrast, B7-H3 knockdown increased both the early and total apoptotic populations compared with sh-NC cells (Fig. 2d). Furthermore, enhanced $\mathrm{Bcl}-2$ expression and reduced Bax and cleaved-caspase 3 expression were observed in B7-H3-overexpressing CRC cells after 4 Gy X-ray irradiation (Fig. 2e and Supplementary Fig. S2c), while downregulated $\mathrm{Bcl}-2$ expression and upregulated Bax and cleaved-caspase 3 expression were observed in B7-H3knockdown CRC cells after 4 Gy X-ray irradiation (Fig. $2 \mathrm{f}$ and Supplementary Fig. S2d). These results demonstrate that $\mathrm{B} 7-\mathrm{H} 3$ protects colorectal cancer cells from irradiation.

\section{B7-H3 blocking antibody 3E8 treatment abrogates B7-H3- mediated CRC cell radioresistance in vivo}

Given that B7-H3 exerts a key effect on CRC radioresistance in vitro, we further used $3 \mathrm{E} 8$, a special $\mathrm{B} 7-\mathrm{H} 3$ blocking antibody that obviously enhanced the radiosensitivity of CRC cells in vitro (Supplementary Fig. S3a), to further determine the role of $\mathrm{B} 7-\mathrm{H} 3$ in regulating $\mathrm{CRC}$ radioresistance. Nude mice bearing empty vector (EV)HCT116 xenografts were treated with 6 Gy or $10 \mathrm{~Gy}$ Xray. As shown in Supplementary Fig. S3b-d, the tumors of mice in the 10 Gy X-ray irradiation treatment group were smaller than those in the 6 Gy X-ray irradiation treatment group. With reference to previous results ${ }^{18,19}$, we choose 10 Gy for the follow-up experiments in vivo.

Nude mice were implanted with EV-HCT116 or B7-H3HCT116 cells and treated with IgG or 3E8 plus 10 Gy Xray radiation (Fig. 3a). Compared with the $\mathrm{EV}+\operatorname{IgG}$ group, the tumors of the $\mathrm{B} 7-\mathrm{H} 3+\mathrm{IgG}$ group showed significant resistance to $10 \mathrm{~Gy} \mathrm{X}$-ray radiation (Fig. 3b-d). Furthermore, 3E8 treatment obviously suppressed the growth of HCT116 xenografts after $10 \mathrm{~Gy} \mathrm{X}$-ray exposure, while 3E8 treatment markedly abrogated the B7-H3mediated radioresistance of HCT116 xenografts (Fig. 3b-d).

Then, the expression of Ki67, a proliferation marker, was examined by immunohistochemistry (IHC) in xenograft tumor tissues. As shown in Fig. 3e, f, the combined treatment with $3 \mathrm{E} 8$ and irradiation significantly reduced the number of Ki67-positive cells in xenografts. The apoptotic cells in the xenograft tissues were also measured with a TUNEL assay. The number of apoptotic cells in the xenograft tissues was significantly increased after the combined treatment with 3E8 and irradiation (Fig. 3g, h). These results together suggest that $\mathrm{B} 7-\mathrm{H} 3$ also contributes to radioresistance of CRC cells in vivo.

\section{B7-H3 promotes KIF15 expression in CRC after irradiation though NF-KB}

To decipher the underlying molecular mechanism associated with B7-H3-mediated radioresistance in CRC cells, RNA sequencing (RNA-seq) was performed to profile the transcriptome changes in B7-H3-knockdown RKO cells after 4 Gy X-ray irradiation. In total, 438 genes with significantly differential expression $(P<0.05)$ in B7H3-knockdown RKO cells were identified. Among these, 208 genes were upregulated, and 230 genes were downregulated (Fig. 4a). A total of 97 genes had a fold change $>2$, and 38 genes had $>2$-fold downregulation (Supplementary Table S1). The top 10 upregulated and downregulated genes are summarized in Fig. 4b. We next validated the results of the RNA-seq analysis with RTqPCR. Consistent with the RNA-seq results, several genes deregulated in the RNA-seq data, including IL1R1, NRF1, STK32C, ZNF329 and UNC5A, were remarkably higher in the sh-B7-H3 group than in the sh-NC group, while the expression of FAM64A, NCOA7, TMUB2, KIF15 and PRDM15 was lower in the sh-B7-H3 group (Supplementary Fig. S4a). We observed that KIF15, a gene encoding a member of the kinesin family of proteins, plays a vital role in regulating the cell cycle ${ }^{20}$. The protein level of KIF15 was upregulated in both $\mathrm{B} 7-\mathrm{H} 3$-overexpressing $\mathrm{CRC}$ cells, while the protein level of KIF15 was downregulated in B7H3-knockdown CRC cells (Fig. 4c and Supplementary Fig. S4b). In 3E8/IgG Ab plus IR treatment xenograft, KIF15 was analyzed by IHC staining. Consistent with the in vitro results, B7-H3 promoted KIF15 expression, while 3E8 treatment reduced the expression of KIF15 (Fig. 4d). These data suggest that B7-H3 could promote KIF15 expression in CRC after IR treatment.

Researches have reported that multiple signaling pathways such as MAPK, NF- $\mathrm{kB}$ and STAT3 were downstream targets of $\mathrm{B} 7-\mathrm{H} 3$ in tumors ${ }^{11,21,22}$. Our previous results have shown that the phosphorylation levels of AKT, NF- $k B$ and STAT3 obviously reduced in CRC cells after B7-H3 knockdown ${ }^{16}$. Moreover, abnormal activation of these signal pathways was involved in radioresistance of tumor $^{23}$. Therefore, we hypothesized that B7-H3 may regulate the expression of KIF15 by affecting one or more signaling pathways. To further investigated our surmise, an STAT3 inhibitor (Cryptonshinone, $4 \mu \mathrm{M}$ ), AKT inhibitor (MK-2206 $2 \mathrm{HCl}, 4 \mu \mathrm{M}$ ), ERK inhibitor (PD98059, $2 \mu \mathrm{M}$ ) and NF- $\mathrm{kB}$ inhibitor (QNZ EVP4593, $2 \mu \mathrm{M}$ ) were used prior to 4 Gy X-ray irradiation. Western blot assays found that B7-H3 regulates the expression level of KIF15 through the NF-kB signaling pathway after IR (Fig. 4e).

\section{The B7-H3/KIF15 axis confers radioresistance in vitro and in vivo}

We hypothesized that B7-H3 may contribute to CRC cell radioresistance by controlling KIF15. To test this 


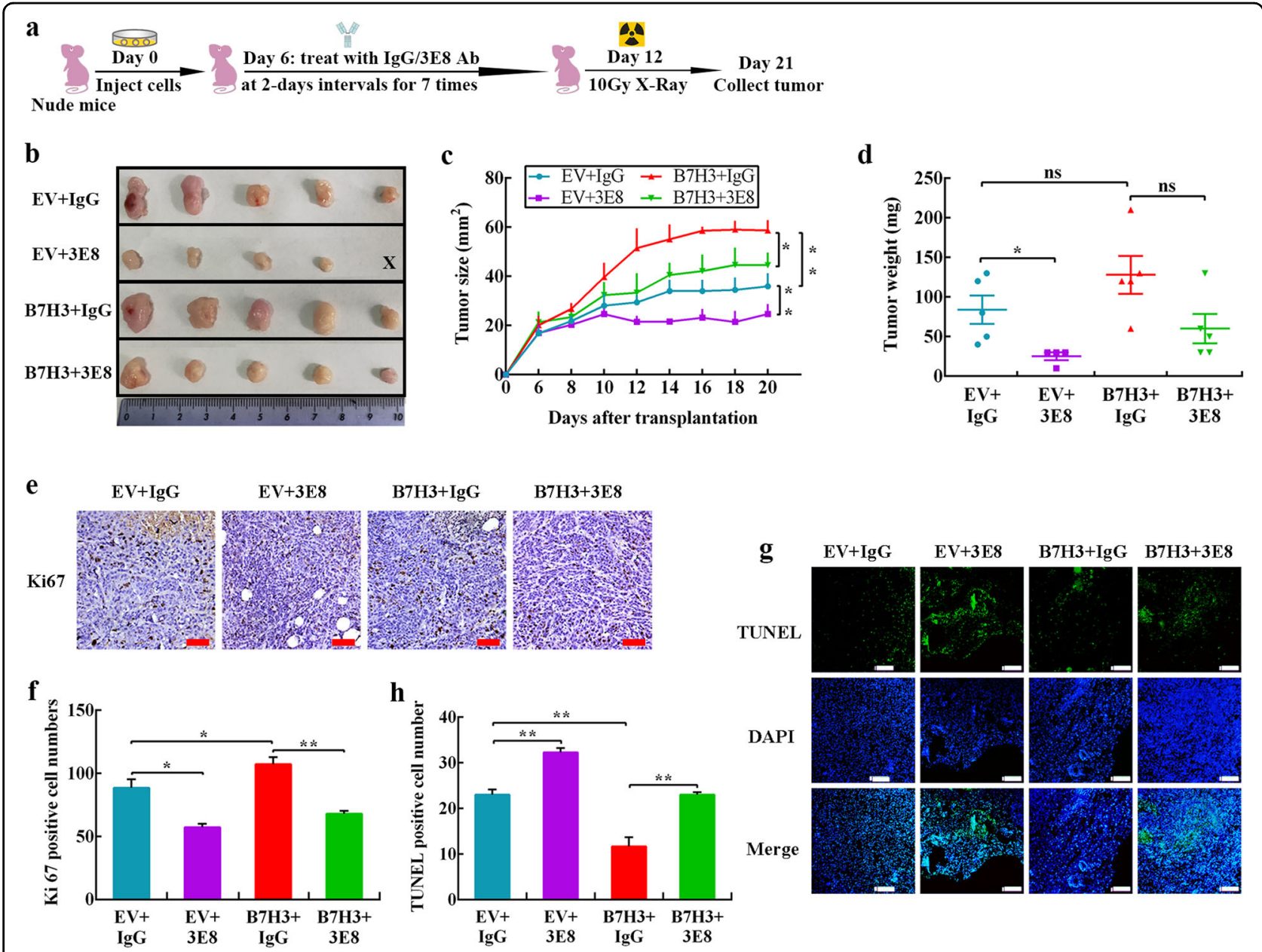

Fig. 3 B7-H3 blocking antibody $3 \mathrm{E} 8$ treatment abrogates B7-H3-mediated CRC cell radioresistance in vivo. a The treatment of each group is shown. HCT116 cells were inoculated under the skin of nude mice. At day 6 after translation, $5 \mathrm{mg} / \mathrm{kg} 3 \mathrm{E} 8 \mathrm{Ab}$ or lgG was intraperitoneally injected into nude mice 7 times for 2-day intervals. On day 12, the xenograft mice were irradiated locally with $10 \mathrm{~Gy}$ X-ray. The tumor size was measured at 2day intervals. b Each group comprised 5 female nude mice. Representative images of tumors formed by EV or B7-H3 cells cotreated with lgG or 3E8 and 10 Gy X-ray irradiation. c The growth curves of tumors formed by the indicated EV or B7-H3 cells cotreated with IgG or 3E8 and 10 Gy X-ray irradiation. $\mathbf{d}$ The weights of tumors formed by the indicated EV or B7-H3 cells cotreated with IgG or $3 \mathrm{E} 8$ and 10 Gy X-ray irradiation. The data are presented as the mean \pm SEM ( $n=5$ mice per group). e, $\mathbf{f ~ K i 6 7 ~ I H C ~ s t a i n i n g ~ i n ~ t u m o r ~ t i s s u e s ~ o f ~ t h e ~ x e n o g r a f t ~ m o d e l ~ w i t h ~ t h e ~ i n d i c a t e d ~ t r e a t m e n t s ~}$ (scale bar, $50 \mu \mathrm{m}) . \mathbf{g}, \mathbf{h}$ TUNEL staining in tumor tissues of the xenograft model with the indicated treatments (scale bar, $50 \mu \mathrm{m}$ ). The data are presented as the mean \pm SEM ( $n=5$ mice per group). ${ }^{* *} P<0.01,{ }^{*} P<0.05$.

hypothesis, an siRNA targeting KIF15, which reduced the expression of KIF15 in CRC cells (Supplementary Fig. S4c, d), or SB743921, a KIF15 inhibitor, was used to treat B7H3-overexpressing CRC cells. The results of cell viability and colony formation assays showed that both KIF15 knockdown and SB743921 treatment abolished the B7$\mathrm{H} 3$-induced increase in radioresistance (Fig. 5a, b and Supplementary Fig. S4e). Additionally, KIF15 knockdown and SB743921 treatment obviously upregulated the apoptotic population of B7-H3-overexpressing CRC cells after 4Gy X-ray irradiation (Fig. 5c and Supplementary Fig. S4f). Moreover, KIF15 knockdown reduced the protein level of $\mathrm{Bcl}-2$, while increased the level of Bax and cleaved-caspase 3 in B7-H3-overexpressing CRC cells after 4Gy X-ray irradiation (Supplementary Fig. S5a). Furthermore, silencing KIF15 and treatment with SB743921 significantly increased the percentage of B7H3-overexpressing CRC cells in S phase after 4 Gy X-ray irradiation (Fig. 5d and Supplementary Fig. S5b). Additionally, KIF15 knockdown increased the protein level of CDK1 in B7-H3-overexpressing CRC cells after 4Gy Xray irradiation (Supplementary Fig. S5c). In summary, these findings reveal that the B7-H3/KIF15 axis contributes to the radioresistance of CRC.

To translate our findings, we assessed whether the B7H3/KIF15 axis conferred resistance to irradiation in vivo. To address whether inhibiting KIF15 activity could reverse $\mathrm{B} 7-\mathrm{H} 3$-mediated CRC cell radioresistance in vivo, 

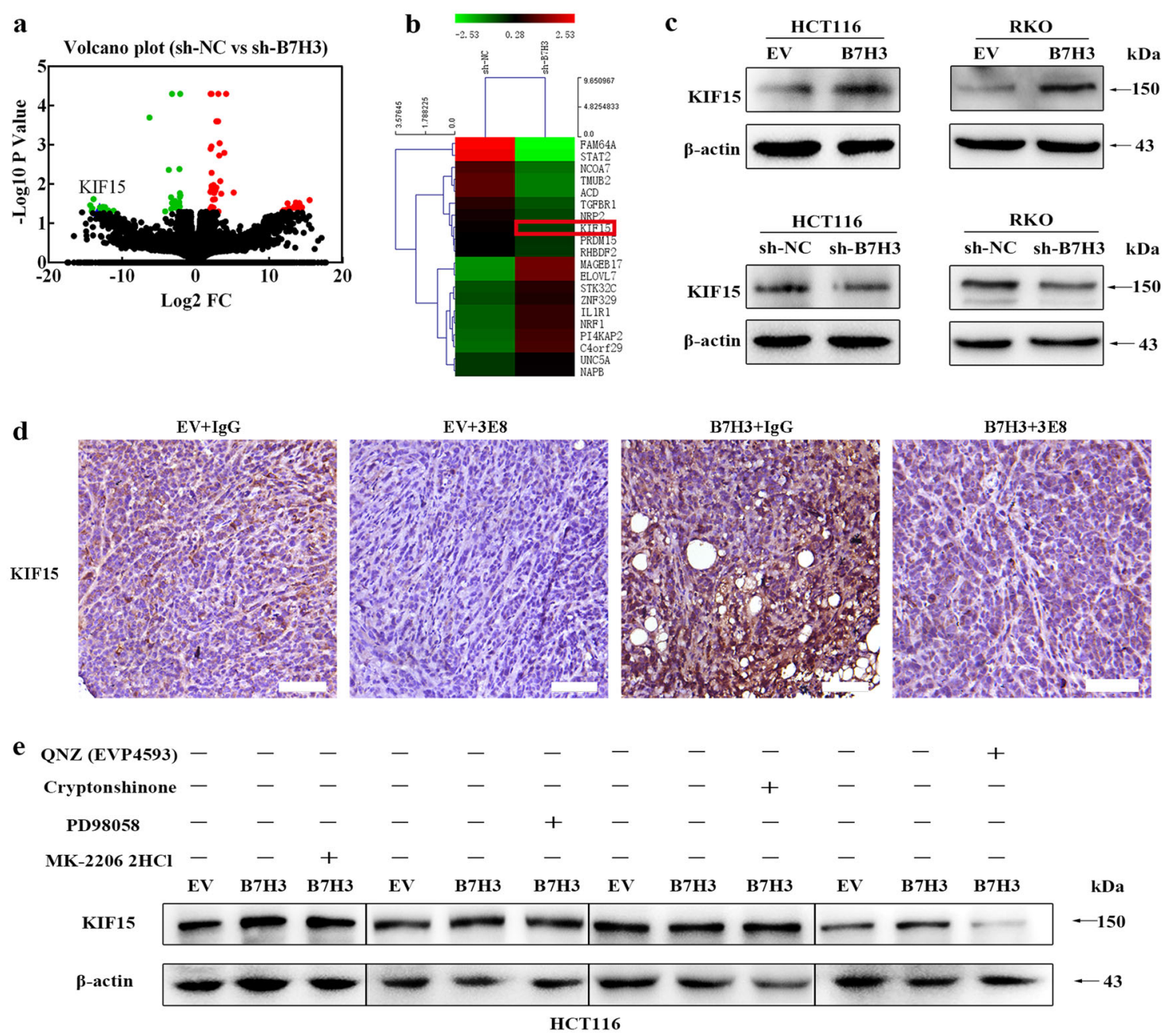

KIF15
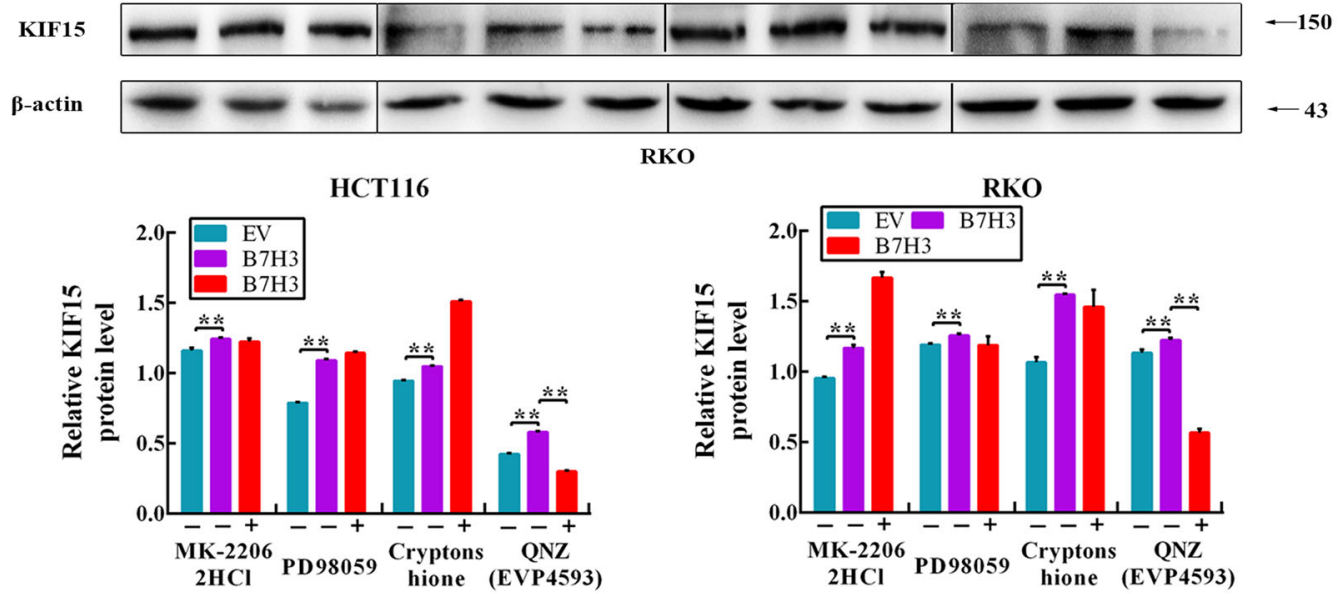

RKO

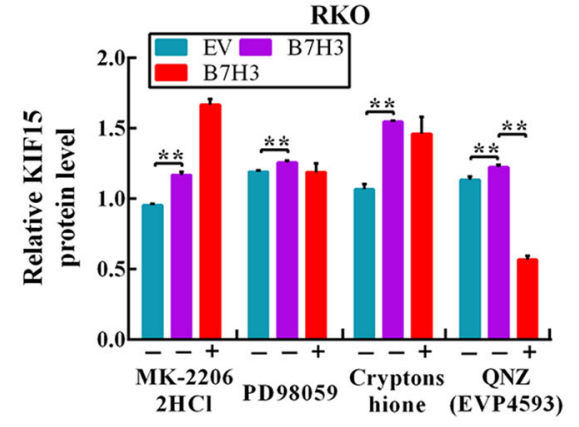

Fig. 4 B7-H3 promotes radioresistance via KIF15. a Total number of genes with significant changes in gene expression $(P<0.05)$. A total of 208 genes were upregulated (red dots), and 230 genes (green dots) were downregulated. $\mathbf{b}$ Heatmap of 20 significantly differentially expressed genes (>2-fold) in sh-B7-H3 versus sh-NC RKO cells. Each column represents a cell number. Each row represents a gene. The red color indicates increased expression. The green color indicates decreased expression. c The protein expression of KIF15 in B7-H3-overexpressing or B7-H3-knockd own CRC cells after exposure to 4 Gy X-ray irradiation. $\beta$-actin served as a loading control. $\mathbf{d}$ One representative image of the IHC analysis of KIF15 protein expression in 3E8/lgG treatment plus IR xenograft tissue sections (scale bar, $50 \mu \mathrm{m}$ ). e The protein expression of KIF15 in B7-H3-overexpressing CRC cells treated with STAT3, AKT, NF-KB, ERK inhibitors prior to treatment with 4 Gy X-ray irradiation. $\beta$-actin served as a loading control. Values are expressed as the mean $\pm \mathrm{SD}$. ${ }^{* *} P<0.01,{ }^{*} P<0.05$. 


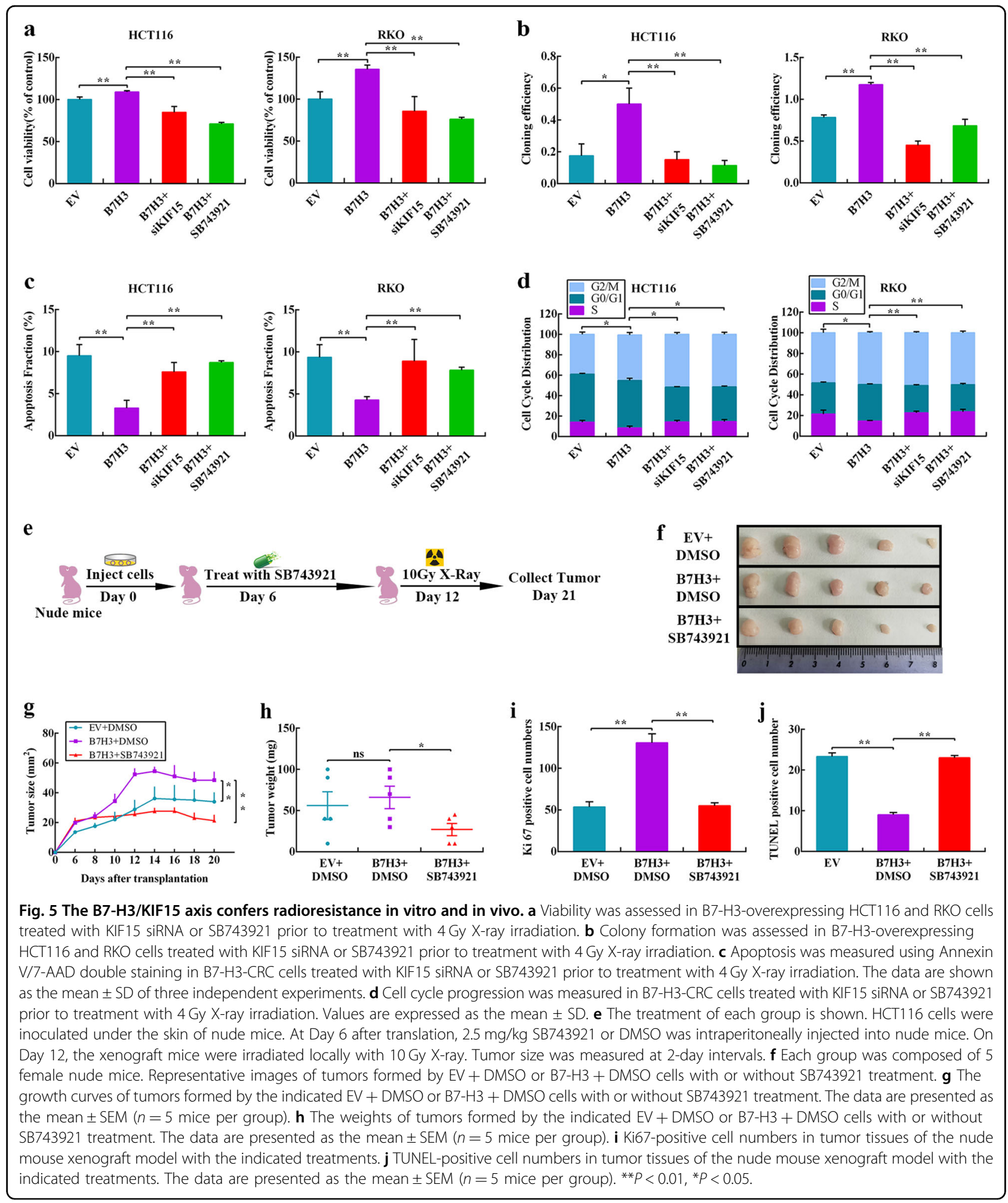

we performed a subcutaneous xenograft tumor assay using B7-H3-HCT116 and respective control cells in athymic nude mice. SB743921 $(2.5 \mathrm{mg} / \mathrm{kg})$ in DMSO was intraperitoneally injected into nude mice on day 6 , and the tumor radioresponse was detected (Fig. 5e). As shown in Fig. $5 \mathrm{f}-\mathrm{h}$, although the tumor weight was not significantly different between the EV + DMSO and B7$\mathrm{H} 3$ + DMSO groups, subcutaneous xenografts in the 
B7-H3 + DMSO group grew significantly faster than those in the EV + DMSO group following 10 Gy X-ray irradiation, whereas cotreatment with SB743921 and irradiation further suppressed tumor growth and tumor weight. Moreover, the expression of KIF15 was increased in $\mathrm{B} 7-\mathrm{H} 3+\mathrm{DMSO}$ than $\mathrm{EV}+\mathrm{DMSO}$ groups, whereas obviously reduced after cotreatment with SB743921 and irradiation (Supplementary Fig. S5d), in accordance with the in vitro data described above. As shown in Fig. 5i and Supplementary Fig. S5d, B7-H3 significantly induced the number of Ki67-positive cells in the xenografts after $10 \mathrm{~Gy}$ X-ray irradiation, while SB743921 treatment significantly reduced the number of Ki67-positive cells. As shown in Fig. 5j and Supplementary Fig. S5e, the number of apoptotic cells in the B7-H3 + DMSO group was significantly decreased relative to that in the $\mathrm{EV}+\mathrm{DMSO}$ group, whereas SB743921 dramatically increased the number of apoptotic cells in xenografts after $10 \mathrm{~Gy}$ X-ray irradiation. These data indicate that $\mathrm{B} 7-\mathrm{H} 3$ promotes radioresistance in vitro and in vivo via KIF15.

\section{The ERK signaling pathway is required for B7-H3/KIF15 axis-mediated radioresistance in CRC cells}

A previous study indicated that KIF15 promotes pancreatic cancer proliferation via the MEK-ERK signaling pathway $^{24}$. More importantly, the ERK signaling pathway plays pivotal roles in radioresistance in many human tumors $^{15}$. Thus, we postulated that the B7-H3/KIF15 axis contributes to radioresistance in $\mathrm{CRC}$ by activating the ERK signaling pathway. To test this hypothesis, we first detected whether the activity of ERK (determined by the ERK DNA-binding activity) was enhanced by the B7-H3/KIF15 axis using luciferase reporter assays. The results showed that $\mathrm{B} 7-\mathrm{H} 3$ overexpression significantly increased ERK activity in HCT116 and RKO cells after IR. In contrast, silencing KIF15 and treatment with SB743921 significantly decreased ERK activity in B7H3-overexpressing CRC cells after 4 Gy X-ray irradiation (Fig. 6a). Meanwhile, the phosphorylation level of ERK in CRC cells after $4 \mathrm{~Gy} \mathrm{X}$-ray irradiation was determined by Western blot analysis. As shown in Fig. 6b, B7-H3 overexpression significantly enhanced the ERK phosphorylation level in CRC cells after 4Gy X-ray irradiation. Conversely, KIF15 knockdown reversed the upregulation of the ERK phosphorylation level in B7-H3overexpressing CRC cells (Fig. 6b and Supplementary Fig. S6a). We further performed immunofluorescence (IF) assay to illustrate that B7-H3/KIF15 axis could upregulate ERK phosphorylation in CRC cells. The expression plasmids carrying human $\mathrm{B} 7-\mathrm{H} 3 \mathrm{cDNA}$ significantly increased the protein levels of B7-H3 in HCT116 and RKO cells (Supplementary Fig. S6b). The results of IF assay showed that B7-H3 overexpression significantly enhanced the fluorescence intensity of both KIF15 and phosphorylated
ERK, wheras KIF15 knockdown counteracted this effect (Fig. 6c). Collectively, these results suggest that the B7H3/KIF15 signaling cascade is involved in the enhancement of radioresistance via the ERK pathway in CRC cells.

To assess whether ERK signaling is key for B7-H3/ KIF15 axis-mediated radioresistance in CRC cells, colony formation, cell viability, cell apoptosis and Western blot assays were performed in B7-H3-overexpressing CRC cells treated with PD98059, an ERK inhibitor, plus 4 Gy $\mathrm{X}$-ray radiation. As shown in Fig. $6 \mathrm{~d}-\mathrm{f}$ and Supplementary Fig. S6c-d, treatment with PD98059 abolished B7-H3induced radioresistance in CRC cells.

\section{Correlation analysis between B7-H3 and KIF15 in CRC tissue samples}

Our previous studies have shown B7-H3 is aberrantly expressed in CRC and contributes to drug resistance ${ }^{7,22}$. To further investigate the correlations between B7-H3 and KIF15 protein levels, we analyzed 123 cancer tissues and adjacent normal tissues of patients with CRC by IHC staining. The results showed that the protein expression levels of both B7-H3 and KIF15 were much higher in cancer tissues than in adjacent normal tissues (Fig. 7a, b). Additionally, it was observed that B7-H3 and KIF15 expression increased with tumor stage. The levels of B7H3 and KIF15 were higher in advanced clinical stages (III and IV) than in early stages (I and II) (Fig. 7a, c and Supplementary Table S2). Pearson's analysis showed a positive correlation between the levels of $\mathrm{B} 7-\mathrm{H} 3$ and KIF15 in CRC patients (Fig. 7d). These results indicate that B7-H3 and KIF15 are positively correlated in human CRC specimens.

\section{Discussion}

Radiotherapy is a common treatment modality for solid tumors, including $\mathrm{CRC}^{3}$. However, radioresistance, resulting in tumor recurrence and a poor prognosis, is still a major reason for treatment failure ${ }^{2}$. Previous studies have indicated that $\mathrm{B} 7-\mathrm{H} 3$ regulates various tumor biological processes, including radioresistance ${ }^{14,25}$. Consistent with these findings, we found that the $\mathrm{B} 7-\mathrm{H} 3$ mRNA and protein expression levels were significantly increased in CRC cells after X-ray irradiation. Moreover, B7-H3 overexpression promoted, while B7-H3 knockdown inhibited, the radioresistance of CRC in vitro and in vivo. Hence, we conclude that B7-H3 may play a critical role in the regulation of radioresistance in CRC cells.

Mounting evidence indicates that immune checkpoint blockade combined with radiotherapy can acquire considerable success in multiple tumor types ${ }^{26-28}$. AntiPD-L1 antibody treatment can improve the response of pancreatic ductal adenocarcinoma to radiotherapy and enhance the effect of radiotherapy in preventing the formation of liver metastases ${ }^{27}$. Given the key 


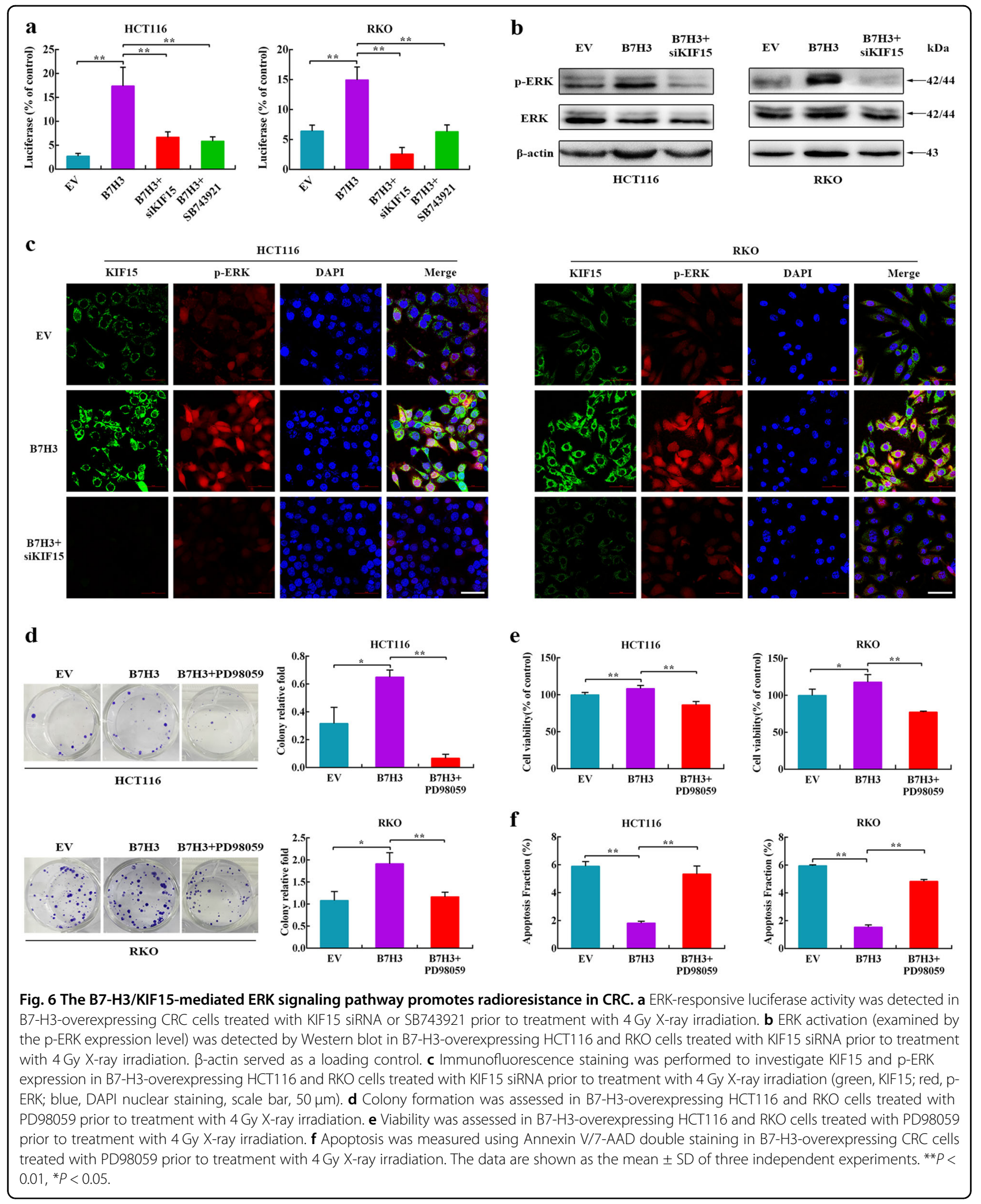




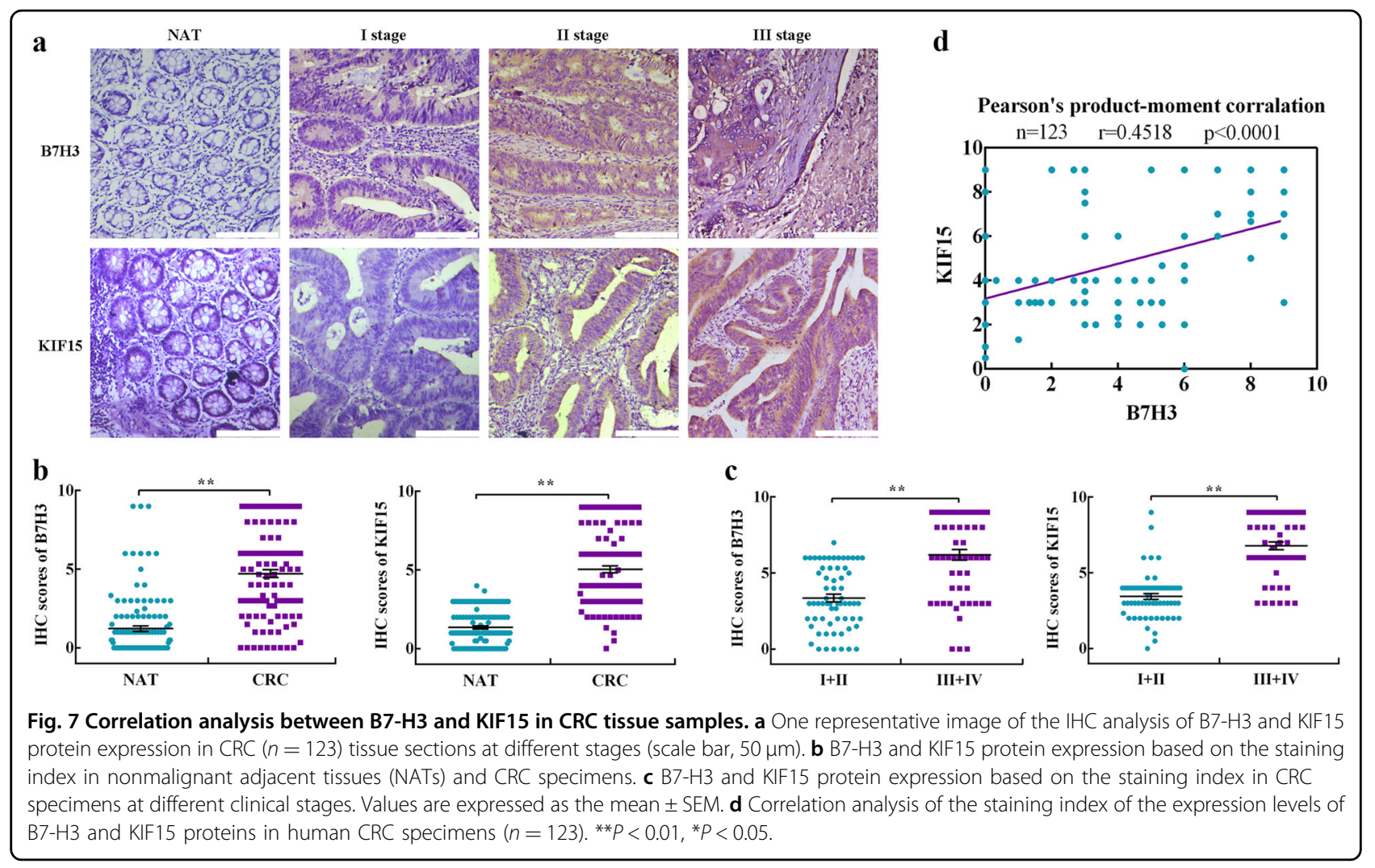

immunologic and nonimmunologic functions of B7-H3 in tumors $^{7,10,14,25}$, it will be valuable to develop blocking antibodies against B7-H3 for tumor therapy. 8H9, which targets $\mathrm{B} 7-\mathrm{H} 3$, combined with craniospinal irradiation has been determined to prolong the survival of patients with metastatic central nervous system neuroblastoma ${ }^{29}$. Moreover, enoblituzumab (MGA271), which directly and specifically targets B7-H3, has also been applied to Phase I trials for different pediatric tumors ${ }^{30}$. In this study, we found that 3E8, a specific B7-H3 blocking antibody, significantly sensitized CRC cells to irradiation in the xenografts of nude mice. Future prospective trials to investigate the effects of 3E8 on the antitumor immune responses of tumors with or without radiotherapy are warranted.

As a critical member of the KIF family, prior studies have noted the importance of KIF15 in the progression of various cancers $^{20,24,31}$. KIF15 is differentially expressed in breast cancer and adjacent tissues, and high levels of KIF15 are significantly correlated with the poor overall survival of patients with breast cancer ${ }^{32}$. KIF15 knockdown induces G1/S phase cell cycle arrest and inhibits cell growth in human lung adenocarcinoma cell lines ${ }^{20}$. In the present study, we found that the expression levels of KIF15 were significantly downregulated in B7-H3knockdown RKO cells after 4Gy X-ray irradiation via RNA-seq analysis. Moreover, the protein level of KIF15 was upregulated when B7-H3 was overexpressed, while the protein level of KIF15 was downregulated in B7-H3knockdown CRC cells after 4Gy X-ray irradiation. Importantly, both KIF15 knockdown and treatment with SB743921, a KIF15-specific inhibitor, reversed the B7-H3induced radioresistant effect in CRC in vitro and in vivo. In addition, we first reported that KIF15 was upregulated in CRC tissue samples compared with normal adjacent tissues and positively correlated with TNM stage. More importantly, there was a positive correlation between the levels of B7-H3 and KIF15 in CRC patients. These results suggest that $\mathrm{B} 7-\mathrm{H} 3$ promotes $\mathrm{CRC}$ radioresistance via KIF15.

Studies have found that NF- $\mathrm{\kappa B}$ is widely present in a variety of cells and induces high expression of inflammatory factors, which is involved in antigen-antibody immune response, inflammation response, cell proliferation and apoptosis ${ }^{33}$. Studies have shown that solar ultraviolet radiation (UVR) significantly increases the expression of PD-L1 molecules in melanocytes, and melanocytes and keratinocytes secrete HMGB1, thereby promoting PD-L1 dependence in melanocytes NF- $\mathrm{kB}$ and IRF3 transcription ${ }^{34}$. Western blot assay found that B7$\mathrm{H} 3$, which was up-regulated after X-ray irradiation, could regulate the expression level of KIF15 through NF-kB. Our previous research also found that B7-H3 can promote the activity of NF- $\mathrm{kB}$ promoter ${ }^{16}$. However, we have 
yet fully understood the specific mechanism by which B7$\mathrm{H} 3$ activates the NF- $\mathrm{kB}$ pathway. Therefore, the manner in which $\mathrm{B} 7-\mathrm{H} 3$ regulates $\mathrm{NF}-\mathrm{kB}$ requires further exploration in future research.

The ERK signaling pathway is well known to play a critical role in several cell processes, including cell cycle progression, cell proliferation, transcription, survival and apoptosis $^{35}$. Above all, the aberrant activation of ERK signaling is involved in radioresistance in several can$\operatorname{cers}^{15}$. The MEK/ERK pathway plays a key role in sustaining the tumorigenicity and in vitro radioresistance of the embryonal rhabdomyosarcoma stem-like cell population $^{36}$. In addition, the ERK pathway has been reported to be involved in mitochondrial ATP-sensitive potassium channel-mediated glioma radioresistance ${ }^{37}$. Consistent with previous results ${ }^{24,38}$, our luciferase reporter assay showed that the B7-H3/KIF15 axis significantly increased ERK activity in CRC cells after 4Gy X-ray irradiation. Western blot and immunofluorescence assays further confirmed the luciferase reporter results. Moreover, treatment with PD98059, an ERK inhibitor, abolished B7H3-induced radioresistance in CRC cells. Therefore, our results suggest that the B7-H3/KIF15 axis protects CRC cells from X-ray irradiation by activating the ERK signaling pathway.

In conclusion, we illustrated that the upregulation of B7-H3 in CRC cells after X-ray irradiation contributes to CRC radioresistance via the KIF15/ERK signaling pathway. Moreover, high KIF15 expression is positively correlated with $\mathrm{B} 7-\mathrm{H} 3$ and TNM stages in CRC tissue samples. Additionally, B7-H3 blockade by 3E8 combined with irradiation significantly controlled tumor growth in xenograft tumor model. Overall, our results indicate that $\mathrm{B} 7-\mathrm{H} 3$ is a potential biomarker that can be used to identify responders for radiotherapy and that the combination of B7-H3 blockade and radiotherapy may improve the therapeutic regimen against CRC.

\section{Acknowledgements}

This study was supported by a grant from the National Natural Science Foundation of China (81672372, 81802843, 81874163, 81372276); Colleges and Universities Natural Science Research Project of Jiangsu Province (19KJB320006, 18KJB320023, 17KJA310004); Suzhou Science \& Technology plan project (SYS2019035); the Defense Basic Research Projects; the Major International (Regional) Joint Research Project (31320103918) and Jiangsu Provincial Medical Youth Talent (QNRC2016770).

\footnotetext{
Author details

'Department of Gastroenterology, The First Affiliated Hospital of Soochow University, 188 Shizi Road, Suzhou, China. ${ }^{2}$ Jiangsu Key Laboratory of Clinical Immunology, Soochow University, 708 Renmin Road, Suzhou, China. ${ }^{3}$ Jiangsu Institute of Clinical Immunology, The First Affiliated Hospital of Soochow University, 708 Renmin Road, Suzhou, China. ${ }^{4}$ Institute of Paediatric Research, Affiliated Children's Hospital of Soochow University, 92 Zhongnan Street, Suzhou, China. Jiangsu Key Laboratory of Gastrointestinal tumor Immunology, The First Affiliated Hospital of Soochow University, 708 Renmin Road, Suzhou, China
}

Conflict of interest

The authors declare that they have no conflict of interest.

\section{Publisher's note}

Springer Nature remains neutral with regard to jurisdictional claims in published maps and institutional affiliations.

Supplementary Information accompanies this paper at (https://doi.org/ 10.1038/s41419-020-03041-4).

Received: 9 June 2020 Revised: 21 September 2020 Accepted: 23 September 2020

Published online: 03 October 2020

\section{References}

1. Siegel, R. L., Miller, K. D. \& Jemal, A. Cancer statistics, 2019. CA Cancer J. Clin. 69 7-34 (2019).

2. Wang, X. C. et al. Genome-wide RNAi screening identifies RFC4 as a factor that mediates radioresistance in colorectal cancer by facilitating nonhomologous end joining repair. Clin. Cancer Res. 25, 4567-4579 (2019).

3. Zhang, $\mathrm{H}$. et al. Inhibition of Notch1/Hes1 signaling pathway improves radiosensitivity of colorectal cancer cells. Eur. J. Pharmacol. 818, 364-370 (2018).

4. Zheng, L. et al. MiR-106b induces cell radioresistance via the PTEN/PI3K/AKT pathways and p21 in colorectal cancer. J. Transl. Med. 13, 252 (2015).

5. Loos, M., Hedderich, D. M., Friess, H. \& Kleeff, J. B7-H3 and its role in antitumor immunity. Clin. Dev. Immunol. 2010, 683875 (2010).

6. Chapoval, A. I. et al. B7-H3: A costimulatory molecule for T cell activation and IFN-y production. Nat. Immunol. 2, 269-274 (2001).

7. Shi, T. et al. B7-H3 promotes aerobic glycolysis and chemoresistance in colorectal cancer cells by regulating HK2. Cell Death Dis. 10, 308 (2019).

8. Zhao, $\mathrm{X}$. et al. Silencing of B7-H3 increases gemcitabine sensitivity by promoting apoptosis in pancreatic carcinoma. Oncol. Lett. 5, 805-812 (2013).

9. Song, J. et al. Epidermal growth factor receptor and B7-H3 expression in esophageal squamous tissues correlate to patient prognosis. Onco. Targets Ther. 9, 6257-6263 (2016).

10. Kang, F. B. et al. B7-H3 promotes aggression and invasion of hepatocellular carcinoma by targeting epithelial-to-mesenchymal transition via JAK2/STAT3/ Slug signaling pathway. Cancer Cell Int. 15, 45 (2015).

11. Zhang, P., Chen, Z., Ning, K., Jin, J. \& Han, X. Inhibition of B7-H3 reverses oxaliplatin resistance in human colorectal cancer cells. Biochem. Biophys. Res. Commun. 490, 1132-1138 (2017).

12. Chen, $\mathrm{C}$. et al. Induced expression of B7-H3 on the lung cancer cells and macrophages suppresses T-cell mediating anti-tumor immune response. Exp. Cell Res. 319, 96-102 (2013).

13. $\mathrm{Li}, \mathrm{Y}$. et al. B7-H3 increases the radioresistance of gastric cancer cells through regulating baseline levels of cell autophagy. Am. J. Transl. Res. 11, 4438-4449 (2019).

14. Pizon, M., Schott, D. S., Pachmann, U. \& Pachmann, K. B7-H3 on circulating epithelial tumor cells correlates with the proliferation marker, Ki-67, and may be associated with the aggressiveness of tumors in breast cancer patients. Int. J. Oncol. 53, 2289-2299 (2018).

15. Marampon, F., Ciccarelli, C. \& Zani, B. M. Biological rationale for targeting MEK ERK pathways in anti-cancer therapy and to potentiate tumour responses to radiation. Int. J. Mol. Sci. 20, 2530 (2019).

16. Wang, R. et al. B7-H3 promotes colorectal cancer angiogenesis through activating the NF-kappaB pathway to induce VEGFA expression. Cell Death Dis. 11, 55 (2020).

17. Liu, M. et al. Heterochromatin protein HP1gamma promotes colorectal cancer progression and is regulated by miR-30a. Cancer Res. 75, 4593-4604 (2015).

18. Xue, J. et al. Activation of PPARalpha by clofibrate sensitizes pancreatic cancer cells to radiation through the Wnt/beta-catenin pathway. Oncogene 37, 953-962 (2018).

19. $\mathrm{Ma}$, H. et al. Icotinib hydrochloride enhances the effect of radiotherapy by affecting DNA repair in colorectal cancer cells. Oncol. Rep. 33, 1161-1170 (2015). 
20. Qiao, Y. et al. Increased KIF15 expression predicts a poor prognosis in patients with lung adenocarcinoma. Cell Physiol. Biochem. 51, 1-10 (2018)

21. Xie, C. et al. Soluble B7-H3 promotes the invasion and metastasis of pancreatic carcinoma cells through the TLR4/NF-kappaB pathway. Sci. Rep. 6, 27528 (2016).

22. Ma, Y. et al. B7-H3 promotes the cell cycle-mediated chemoresistance of colorectal cancer cells by regulating CDC25A. J. Cancer 11, 2158-2170 (2020).

23. Rodriguez-Ruiz, M. E., Vitale, I., Harrington, K. J., Melero, I. \& Galluzzi, L. Immunological impact of cell death signaling driven by radiation on the tumor microenvironment. Nat. Immunol. 21, 120-134 (2020).

24. Wang, J., Guo, X., Xie, C. \& Jiang, J. KIF15 promotes pancreatic cancer proliferation via the MEK-ERK signalling pathway. Br. J. Cancer 117, 245-255 (2017).

25. Parker, A. S. et al. Evaluation of B7-H3 expression as a biomarker of biochemical recurrence after salvage radiation therapy for recurrent prostate cancer. Int. J. Radiat. Oncol. Biol. Phys. 79, 1343-1349 (2011).

26. Deng, L. et al. Irradiation and anti-PD-L1 treatment synergistically promote antitumor immunity in mice. J. Clin. Invest. 124, 687-695 (2014).

27. Azad, A. et al. PD-L1 blockade enhances response of pancreatic ductal adenocarcinoma to radiotherapy. EMBO Mol. Med. 9, 167-180 (2017).

28. Zeng, J. et al. Anti-PD-1 blockade and stereotactic radiation produce longterm survival in mice with intracranial gliomas. Int. J. Radiat. Oncol. Biol. Phys. 86, 343-349 (2013).

29. Kramer, K. et al. Compartmental intrathecal radioimmunotherapy: results for treatment for metastatic CNS neuroblastoma. J. Neurooncol. 97, 409-418 (2010).
30. Picarda, E., Ohaegbulam, K. C. \& Zang, X. Molecular pathways: targeting B7-H3 (CD276) for human cancer immunotherapy. Clin. Cancer Res. 22, 3425-3431 (2016).

31. Zou, J. X. et al. Kinesin family deregulation coordinated by bromodomain protein ANCCA and histone methyltransferase MLL for breast cancer cell growth, survival, and tamoxifen resistance. Mol. Cancer Res. 12, 539-549 (2014).

32. Song, $X$. et al. Distinct diagnostic and prognostic values of kinesin family member genes expression in patients with breast cancer. Med. Sci. Monit. 24, 9442-9464 (2018).

33. Bastian, Hoesel \& Schmid, J. A. The complexity of NF-KB signaling in inflammation and cancer. Mol. Cancer 12, 86 (2013).

34. Wang, W. et al. Upregulation of PD-L1 via HMGB1-activated IRF3 and NFkappaB contributes to UV radiation-induced immune suppression. Cancer Res 79, 2909-2922 (2019).

35. Najafi, M., Ahmadi, A. \& Mortezaee, K. Extracellular-signal-regulated kinase/ mitogen-activated protein kinase signaling as a target for cancer therapy: an updated review. Cell Biol. Int. 43, 1206-1222 (2019).

36. Ciccarelli, $C$. et al. Key role of MEK/ERK pathway in sustaining tumorigenicity and in vitro radioresistance of embryonal rhabdomyosarcoma stem-like cell population. Mol. Cancer 15, 16 (2016).

37. Huang, L. et al. Mitochondrial KATP channels control glioma radioresistance by regulating ROS-induced ERK activation. Mol. Neurobiol. 52, 626-637 (2015)

38. Zhao, H. et al. KIF15 promotes bladder cancer proliferation via the MEK-ERK signaling pathway. Cancer Manag. Res. 11, 1857-1868 (2019). 